\title{
SUSCEPTIBILITY TO INTERGRANULAR CORROSION OF AA2024 ALUMINUM ALLOY USED IN AEROSPACE INDUSTRY
}

\author{
Vasile HOTEA \\ Technical University of Cluj Napoca - North University Center of Baia Mare \\ e-mail: vasilehotea50@yahoo.com
}

\begin{abstract}
Idea in this work has been performed the susceptibility to intergranular corrosion of high strength aluminum alloy 2024 in aggressive corrosion environments. Intergranular corrosion is limited to the immediate area of the grain boundary and is not apparent by simple visualization and the resistance to intergranular corrosion of aluminum alloys in the $\mathrm{Al}-\mathrm{Cu}-\mathrm{Mg}$ system is related to the heating conditions applied during the solution treatment. This is can be an important source of limiting the life of the aircraft body where alloys from the 2xxx series are used, which is why they are systematically subjected to intergranular corrosion tests in dedicated laboratories.
\end{abstract}

KEYWORDS: AA2024 aluminum alloy, intergranular corrosion, metallography, optical microscopy

\section{Introduction}

The 2024 Al alloy (AA2024) is widely used in aviation industry due to its high strength to weight ratio, its high mechanical properties resulting from alloying elements such as copper [1, 2]. However, alloying elements generate a heterogeneous microstructure leading to an increase of the susceptibility of the alloy to localized corrosion such as intergranular corrosion [3].

The susceptibility to intergranular corrosion (IGC) depends mainly on the alloy composition and the thermal treatment applied. This form of corrosion can be very dangerous because the attack can be undetectable through the material and can damage the mechanical properties of the metal and cause rupture without visible external corrosion alloys. In the presence of seawater, the phenomenon of corrosion can occur, which consists in an attack on the anode area at the grain boundary. This attack may be relatively rapid and may exist without visible evidence [4].

The microstructure of metals and alloys is made up of grains, separated by grain boundaries. Intergranular corrosion is localized attack along the grain boundaries, or immediately adjacent to grain boundaries, while the bulk of the grains remain largely unaffected. This form of corrosion is usually associated with chemical segregation effects (impurities have a tendency to be enriched at grain boundaries) or specific phases precipitated on the grain boundary [5].

The type of treatment according to the ANSI H35 standard and the definitions used in the United States and Europe, through the European standard EN 515 and internationally through the standard ISO 2107, standard used for heat treated aluminum alloys is temper T3 and means solution treatment followed by natural aging and during the heat treatment, the aluminum alloy (AA2024-T3) is heated to a temperature at which the alloying elements dissolve [6].

The AA2xxx series of alloys are among the most complicated to analyse. While there have been several reports of the compositions of different phases within this group, most have focused on the legacy alloy AA2024-T3, which, unfortunately, is one of the most complex of the $2 x x x$ series of alloys. To begin to understand the microstructure and its influence on corrosion it is important to know compositions of second phase intermetallic particles. Heat treatment dissolves mainly much of the $\mathrm{Al}_{2} \mathrm{Cu}$ and $\mathrm{Al}_{2} \mathrm{CuMg}$ [7].

Boag et al. showed that only the most intermetallic compounds on the surface of AA2024T3 were associated with chloride signals drawing a strong link between clustering and stable pitting. The subsurface attack at these sites was almost exclusively intergranular, penetrating as much as 60 $\mu \mathrm{m}$ in 120 minutes exposure to $0.1 \mathrm{M} \mathrm{NaCl}[7,8]$. 
Standard Practice for Evaluating Intergranular Corrosion Resistance of Heat Treatable Aluminum Alloys by Immersion in alkaline environments is ASTM G110-92 (2009) [9].

The purpose of this paper was to obtain more complete and clear information on the influence of the corrosion medium on the 2024-T3 alloy and also the influence of microstructure and heat treatment on the corrosion resistance. The corrosion behaviour was evaluated by intergranular corrosion tests, regarding the ASTM standard G110 - 92 for the evaluation of intergranular corrosion resistance of the thermally treated aluminum alloys by immersion in sodium chloride solution and hydrogen peroxide.

\section{Experimental procedure}

\subsection{Materials and method}

In this paper, the aluminum alloy 2024 - T3 extruded according to the classification of the American Aluminum Association was considered. This high strength alloy is commonly used in the aerospace industry and samples was received from UAC Europe SRL (Romania). The composition of the alloy is shown in Table 1.

Table 1. Chemical composition of extruded aluminum alloy 2024 T3 [10]

\begin{tabular}{|c|c|c|c|c|c|c|c|c|c|c|}
\hline Element & $\mathrm{Al}$ & $\mathrm{Mg}$ & $\mathrm{Si}$ & $\mathrm{Mn}$ & $\mathrm{Cu}$ & $\mathrm{Zn}$ & $\mathrm{Fe}$ & $\mathrm{Cr}$ & $\mathrm{Ti}$ & Other \\
\hline (\% w.) & Balance & $1.2-1.8$ & 0.5 & $0.3-0.9$ & $3.8-4.9$ & 0.25 & Max 0.5 & 0.10 & 0.15 & 0.15 \\
\hline
\end{tabular}

As can be seen from Table 1, the main alloying element for alloy 2024-T3 is copper with magnesium that plays an important role in this alloy class.

For the IGC corrosion test according to ASTM G110-92, a sample of the previous month is taken from the largest thickness extrusion profile $(<0.25$ "). The preparation of samples is shown schematically in Figure 1.

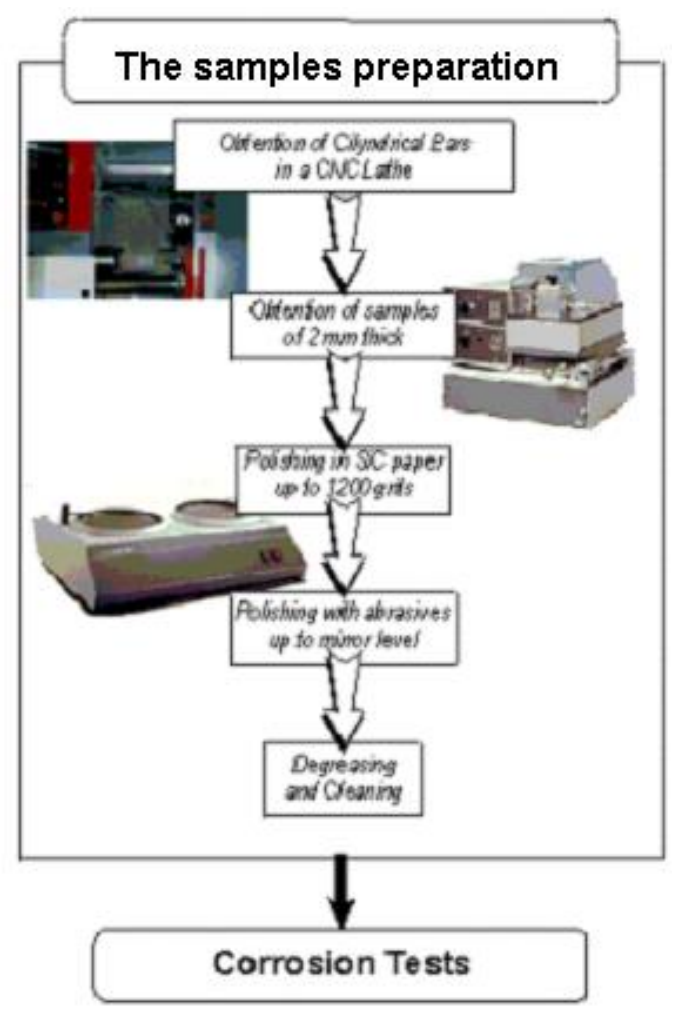

Fig. 1. The samples preparation
Samples must have clean surfaces, free of impurities, oils or any other kind of residue. Removal was performed by immersion of the sample in a $5 \%$ sodium hydroxide solution and $60{ }^{\circ} \mathrm{C}$ temperature, followed by immersion for $1 \mathrm{~min}$ in a concentrated nitric acid solution.

The samples exposed in the corrosion solution should be carried out at $30 \pm 3{ }^{\circ} \mathrm{C}$. The samples were identified in such a way as to ensure the traceability of the test material. The test specimen was machined by grinding / milling so that $10 \%$ of the test surface was removed. All solutions used for the test are prepared using chemical reagents and deionized water. Prior to intergranular corrosion testing, the samples were degreased by immersion for one minute in the degreasing solution.

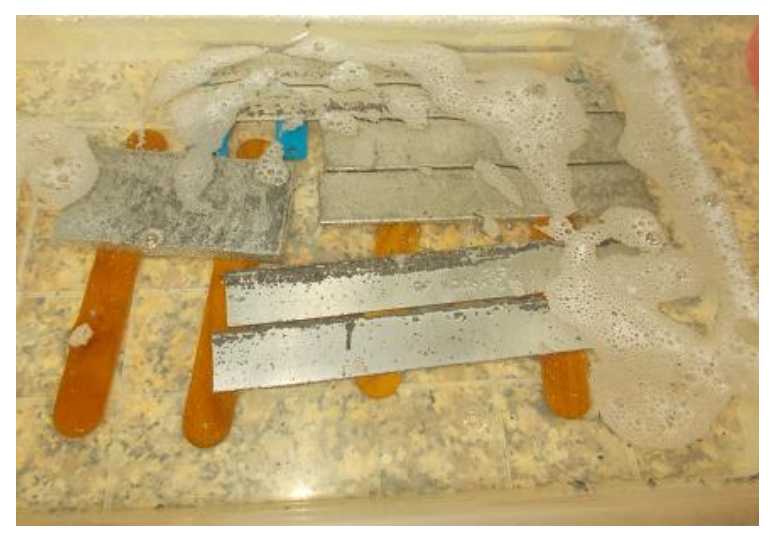

Fig. 2. Place the samples in the vessel with the corrosion solution

The intergranular corrosion test solution consisted of 57 grams of sodium chloride $(\mathrm{NaCl})$, 
oxygenated water $\left(\mathrm{H}_{2} \mathrm{O}_{2}\right) 10 \mathrm{~mL}$ and deionized water $945 \mathrm{~mL}$.

In the case of the use of several samples, they will be immersed in the same vessel with the corrosive solution, as seen in Figure 2, taking care to be arranged separately so that they do not touch each other. The container in which the samples are to be tested must be made of plastic or glass.

\section{Optical microscopy}

The corroded samples were embedded and analysed metallographically on a Leica DM2500 optical microscope, with $100 \mathrm{~W}$ bright illumination, high quality, optical performance, as well as lastminute accessories.

It is coupled to a Windows operating system, determining the microstructure of the sample surface,

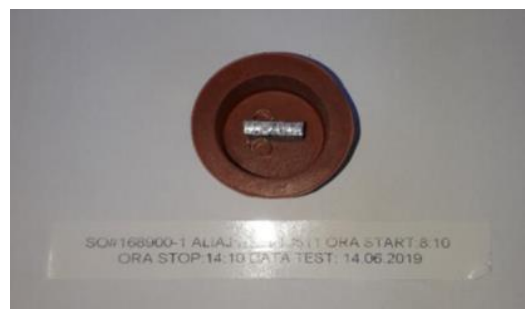

Fig. 3. Sample taken (left) and embedded in cross section (right)

\subsection{Metallographic evaluation}

The procedure for the metallographic evaluation of corrosion damage was in accordance to ASTM G46-94 (2018) [11]. The maximum depth of intergranular corrosion is investigated in the cross section by examination under the optical microscope, after having previously been pre-treated by grinding, polishing and chemical attack with $5 \%$ orthophosphoric acid for 5 minutes. After a 6-7 hour immersion, the specimens are removed from the corrosive solution, washed, brushed with a soft brush to remove corrosion products and dried with compressed air. Each specimen is examined at a magnification of 10x for the location of the corroded surface.

After locating the corroded surface, it is cut from the corroded sample at a distance of approximately $20 \mathrm{~mm}\left(3 / 4^{\prime \prime}\right)$ from the edge length. The samples thus taken are prepared for the assessment of susceptibility to intergranular corrosion (IGC). The sample preparation is done according to the laboratory methodology, and the image of the corroded and clean samples are shown in Figure 3.
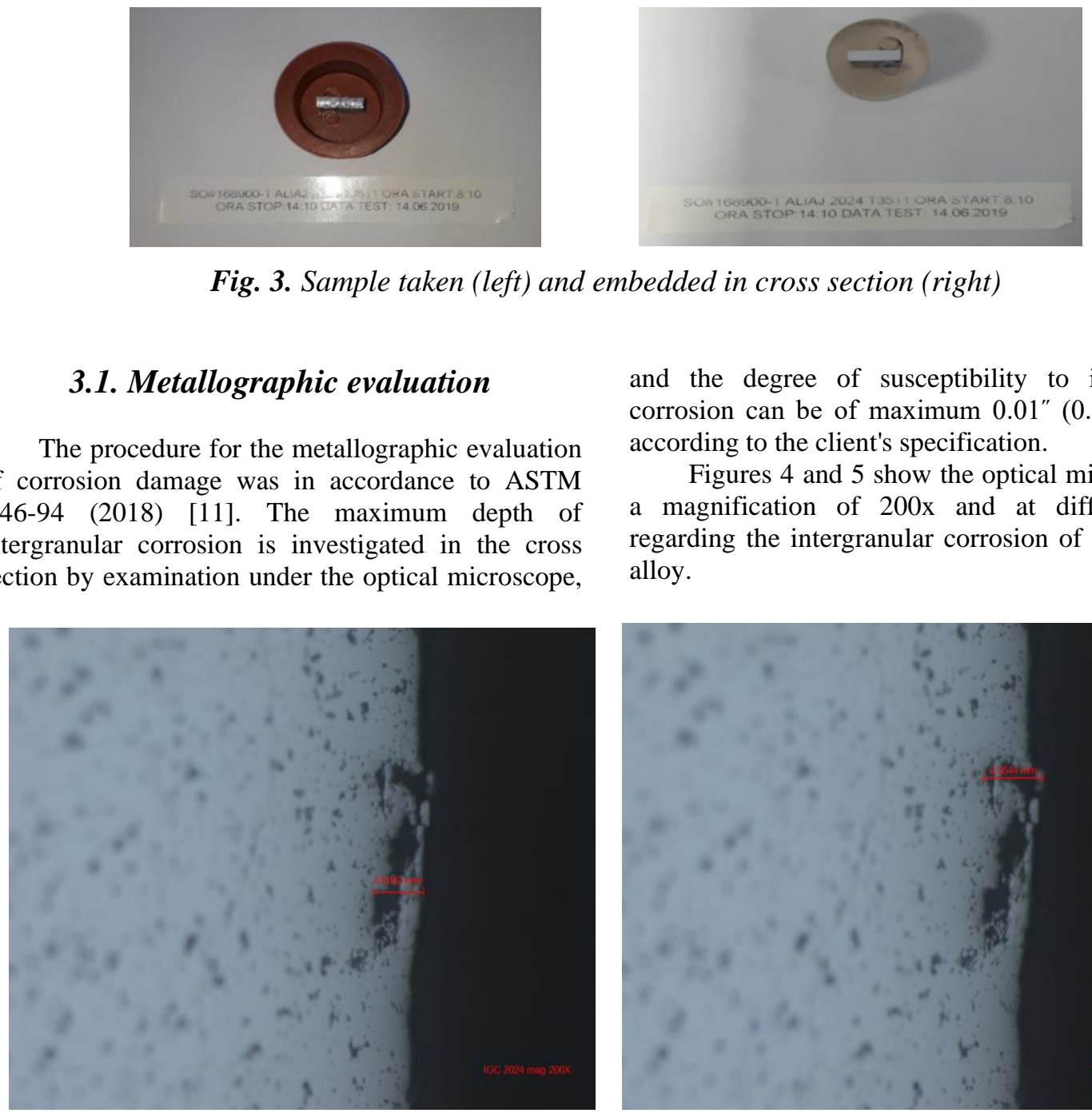

and the degree of susceptibility to intergranular corrosion can be of maximum $0.01 "(0.254 \mathrm{~mm})$ or according to the client's specification.

Figures 4 and 5 show the optical micrographs at a magnification of 200x and at different times regarding the intergranular corrosion of the 2024-T3 alloy.

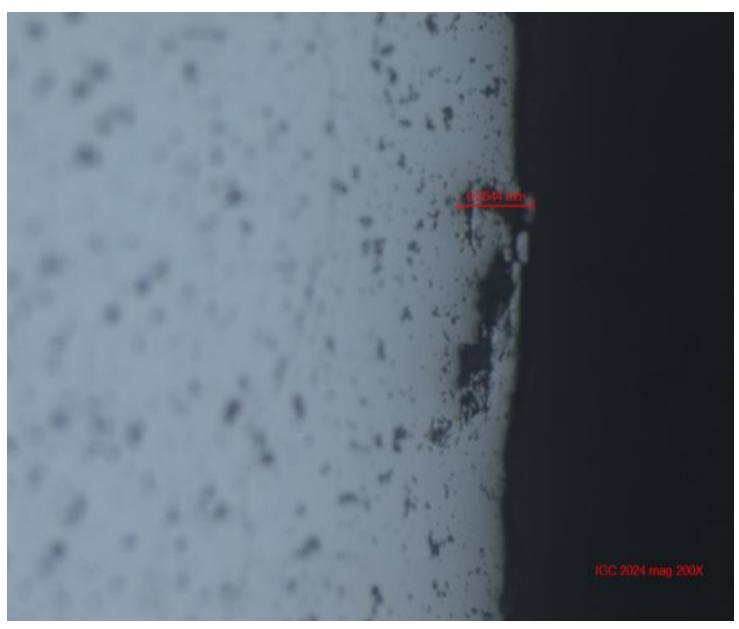

Fig. 4. Corrosion after 2 hours (left) and Corrosion after 4 hours (right) 

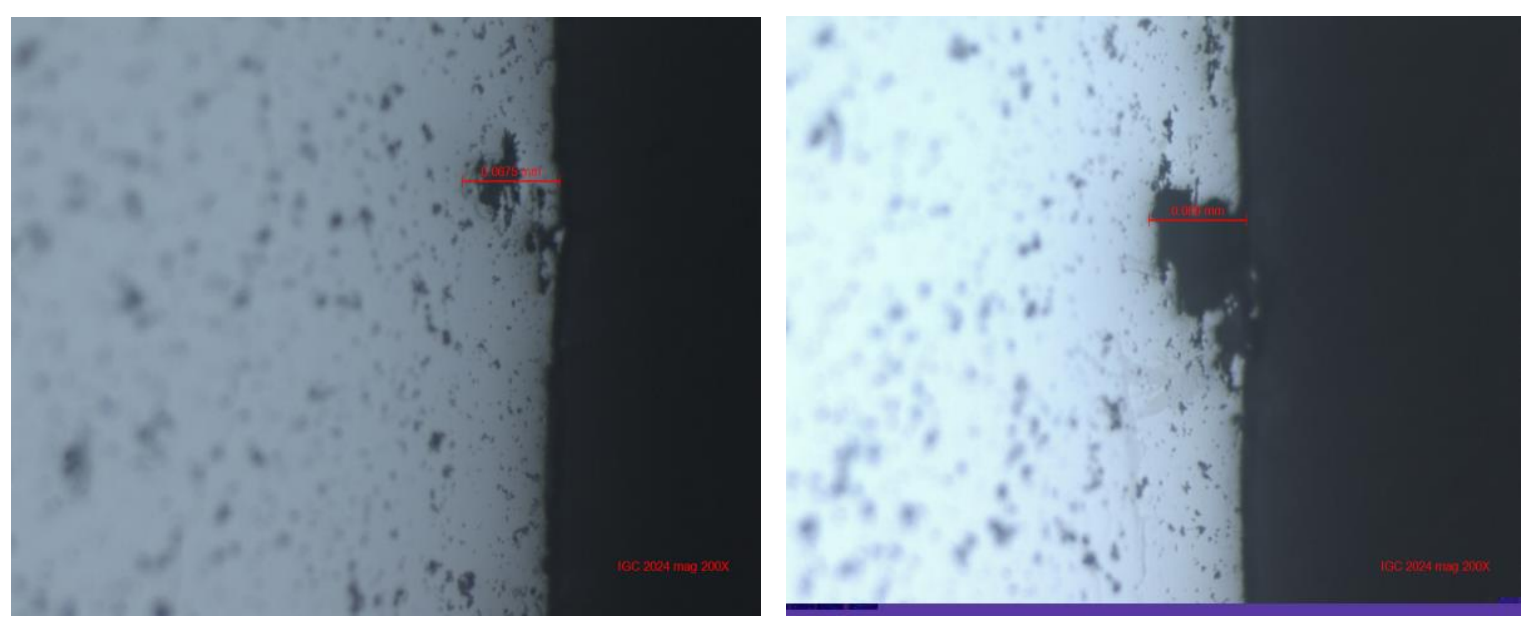

Fig. 5. Corrosion after 6 hours (left) and Corrosion after 8 hours (right)

After 4 hours of exposure, even if the average depth of corrosion increased slightly to $0.054 \mathrm{~mm}$, most of the developed corrosion points are still a little shallow, where their distribution is observed in terms of their depth.
The results regarding the metallographic examination on the depth of the samples are centralized in Table 2 .

Table 2. Metallographic examination of the corrosion samples on their depth

\begin{tabular}{|c|c|c|c|c|}
\hline Exposure time $[\mathrm{h}]$ & 2 & 4 & 6 & 8 \\
\hline $\begin{array}{c}\text { Characterization of } \\
\text { corrosion }\end{array}$ & Pits & Pits & $\begin{array}{c}\text { Intergranular } \\
\text { corrosion }\end{array}$ & Exfoliation corrosion \\
\hline
\end{tabular}

The metallographic evaluation of the corrosion on the top surface is summarized in Table 2 and the corrosion damage evolution in Figure 4.-5. The results indicate that corrosion damage evolves from pitting to exfoliation progressively.

By increasing the corrosion exposure time from 6 hours to 8 hours, the pits become deeper and wider, and the cross sections have shown that the most pronounced shape is mainly quoted at $0.069 \mathrm{~mm}$ leading to a stratification and exfoliation which is a severe form of intergranular corrosion.

In extruded products, if the crystalline grains are flattened and elongated in the extrusion direction, the presence of intergranular corrosion can lead to stratification and exfoliation, as seen in Figure 6.

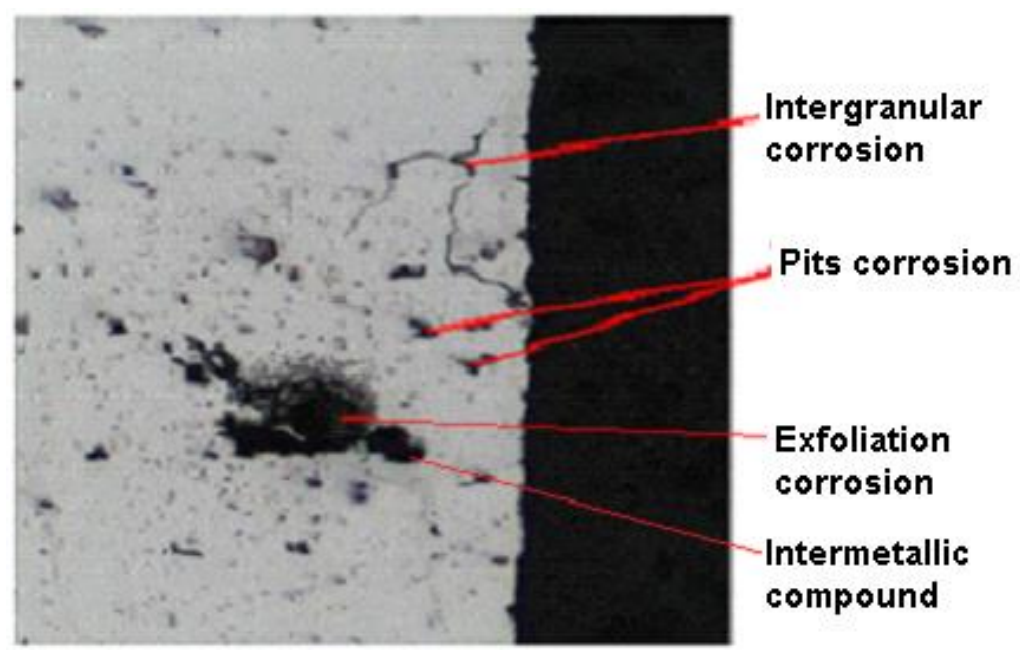

Fig. 5. Intergranular corrosion at 2024-T3 alloy (500x) 
From a corrosion perspective, the dominant characteristics of the high strength alloy microstructure of the $2 \mathrm{xxx}$ series are the granular structure and the distribution of the intermetallic compounds $\left(\mathrm{Al}_{3} \mathrm{Fe}, \mathrm{Al}_{2} \mathrm{Cu}\right)$, which include components and particles of impurities and dispersoids that precipitate. On a larger scale, the corrosion is observed around the constituents and impurities of the particles that result from a severe corrosion attack by points as observed in the above image [12, 13].

The high-strength 2024 -T3 alloy has a phase distribution ranging in size from about 0.1 microns to a few nanometres in diameter. These particles (as the secondary phase) are enriched in different alloying elements, particles that form during the initial solidification of the moulded blank and do not dissolve to a large extent during the heat treatment. This is why aluminum alloys with copper content (from the 2xxx series), with heat treatments in temper $\mathrm{T} 3$, are susceptible to intergranular corrosion.

\section{Conclusions}

In this paper, an extensive metallographic investigation was carried out to evaluate the corrosion damage of the aluminum alloy AA2024-T3 after exposure to corrosion in strongly corrosive environments with $\mathrm{NaCl}$ solution and oxygenated water $\left(\mathrm{H}_{2} \mathrm{O}_{2}\right)$. The defects were quantified in terms of the metallographic characteristics of the corrosion, namely the depth of the corrosion points and their thickness.

The susceptibility to intergranular corrosion depends mainly on the composition of the alloy and the heat treatment applied. This form of corrosion can be very dangerous, as the attack can go undetected in the material and cause cracking and tearing, with no visible signs of corrosion in the alloy.

Corrosion damage has been found to progressively evolve, from pits to intergranular corrosion and exfoliation, with exposure time.
The cross sections of the embedded samples showed that the lateral surfaces of the material accumulated a significant amount of deep sample damage compared to the damage detected on the surface of the samples.

As a result, this can be an important source of limiting the life of the aircraft body where alloys from the $2 \mathrm{xxx}$ series are used, which is why they are systematically subjected to intergranular corrosion tests in dedicated laboratories.

\section{References}

[1]. ***, Aging of U.S. AirForce Aircraft: Final Report, Commission on Engineering and Technical Systems, Committee on Aging of U.S. Air Force Aircraft, National Research Council, Division on Engineering and Physical Sciences and National Materials Advisory Board, National Academies Press, Washington, 1997.

[2]. ***, Success stories: air force; material substitution and new sealing, vol. 7, no. 4, 2003.

[3]. M.-L. de Bonfils Lahovary et al., Corrosion Science, 119, p. 60-67, 2017.

[4]. Roberge P. R., Handbook of corrosion engineering, NewYork. McGraw-Hill, Inc. 2000.

[5]. $\quad$ ***, https://www.nace.org/resources/generalresources/corrosion-basics/group-2/intergranular-corrosion.

[6]. ***, American National Standard Alloy Temper Designation System for Aluminum, Secretariat, The Aluminum Association, Inc., ANSI H35.1/H35.1(M) - 2009 Revision of H35.1/H35.1(M) 2006approved April 7, 2009.

[7]. Zaki Ahmad, Recent Trends in Processing and Degradation of Aluminium Alloys, Chapter 10 - High Strength Al-Alloys: Microstructure, Corrosion and Principles of Protection, ISBN: 978953-307-734-5, DOI: 10.5772/741, 2011.

[8]. Boag A., A. E. Hughes, et al., Corrosion of AA2024-T3 Part I. Localised corrosion of isolated IM particles, Corrosion Science, 53(1), p. 17-26, 2011.

[9]. ***, Standard Practice for Evaluating Intergranular Corrosion Resistance of Heat Treatable Aluminium Alloys by Immersion in Sodium Chloride and Hydrogen Peroxide Solution, ASTM International, West Conshohocken, PA, ASTM G110-92, 2009.

[10]. ***, http://asm.matweb.com.

[11]. ***, Standard Guide for Examination and Evaluation of Pitting Corrosion, DOI: 10.1520/G0046-94R18, ASTM G46-94, 2018.

[12]. Vargel C., Jacques M., Schmidt M. P., Types of Corrosion on Aluminium, in Corrosion of Aluminium, Elsevier: Amsterdam. p. 113-146, 2004.

[13]. Glenn M., Muster T. H., et al., Corrosion of AA2024-T3 Part III: Propagation, Corrosion Science, 53(1), p. 40-50, 2011. 